WellBeing International

WBI Studies Repository

$6-2004$

\title{
An Evaluation of the US High Production Volume (HPV) Chemical- testing Programme: A Study in (Ir)Relevance, Redundancy and Retro Thinking
}

Andrew Nicholson

Jessica Sandler

People for the Ethical Treatment of Animals

Troy Seidle

People for the Ethical Treatment of Animals

Follow this and additional works at: https://www.wellbeingintlstudiesrepository.org/acwp_arte

Part of the Animal Experimentation and Research Commons, Animal Studies Commons, and the Other Business Commons

\section{Recommended Citation}

Nicholson, A., Sandler, J., \& Seidle, T. (2004). An evaluation of the US High Production Volume (HPV) chemical-testing programme: a study in (ir) relevance, redundancy and retro thinking. ATLA-

NOTTINGHAM-, 32, 335-342.

This material is brought to you for free and open access by WellBeing International. It has been accepted for inclusion by an authorized administrator of the WBI Studies Repository. For more information, please contact wbisr-info@wellbeingintl.org.

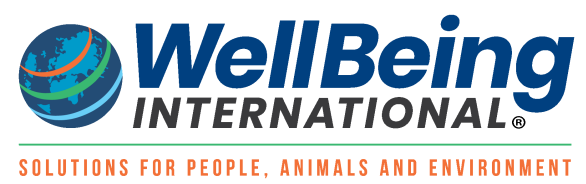




\title{
An Evaluation of the US High Production Volume (HPV) Chemical-testing Programme: A Study in (Ir)Relevance, Redundancy and Retro Thinking
}

\author{
Andrew Nicholson, ${ }^{1}$ Jessica Sandler ${ }^{2}$ and Troy Seidle ${ }^{2}$ \\ 1Independent Consultant; 2 People for the Ethical Treatment of Animals, Norfolk, VA, USA
}

\begin{abstract}
Summary - Under the US Environmental Protection Agency (EPA) High Production Volume (HPV) Challenge Programme, chemical companies have volunteered to conduct screening-level toxicity tests on approximately 2800 widely-used industrial chemicals. Participating companies are committed to providing available toxicity information to the EPA and presenting testing proposals for review by the EPA and posting on the EPA Web site as public information. People for the Ethical Treatment of Animals (PETA) and a coalition of animal protection organisations have reviewed all the test plans submitted by the participating chemical companies for compliance with the original HPV framework, as well as with animal welfare guidelines issued by the EPA in October 1999. Our review found major and recurring flaws in the programme's execution, as well as in its fundamental design. Approximately $75 \%$ of the test plans reviewed violated fundamental terms of the programme. Many participating companies failed to conduct comprehensive analyses of available data and instead proposed superfluous and meaningless tests. The US HPV programme's exclusion of human health and exposure data has led to numerous examples of irrelevant experiments that will not affect how a chemical substance is used or handled. Contrary to claims by both the EPA and Environmental Defense that few new animal tests are being performed, an estimated 100,000 animals have already died in this US Government-sponsored animal-testing programme.
\end{abstract}

Key words: animal testing, animal welfare, category testing, chemical screening, chemical testing, EPA, exposure assessment, hazard assessment, high production volume, HPV, OECD, SIDS.

Address for correspondence: J. Sandler, People for the Ethical Treatment of Animals, 501 Front Street, Norfolk, VA 23510, USA.

E-mail: jessicas@peta.org

\section{Introduction}

The USA defines high production volume (HPV) chemicals as those which are manufactured, sold, or imported in volumes of one million pounds or more per year. In response to pressure from the advocacy group, Environmental Defense (ED), which asserted that there was a "virtual vacuum of information" on the 2800 most widely used chemicals in the USA (1), the US Environmental Protection Agency (EPA) entered into a series of closed-door meetings with ED and the American Chemistry Council (ACC), the product of which was the HPV Challenge Programme. According to the EPA, the goal of the HPV programme is to "prioritise chemicals for further testing" (2) by generating basic toxicity data in line with the OECD "screening information data set" (SIDS; 3). This is a screening level testing programme employing, as ED stated, "dumbed-down toxicology" (1). Despite the fact that upwards of 1400 animals may be killed for each chemical that undergoes a full SIDS battery of tests (4), most US animal protection organisations were never notified of the existence and development of the HPV programme.

One of the major assumptions underlying the HPV programme is that chemicals produced in large volumes pose a greater hazard, on average, than other substances. However, Cunningham \& Rosenkranz (5) have documented, by using computerised structure-activity relationship analysis, that "for all toxic effects (including mutagenicity, genotoxicity, carcinogenicity, and developmental toxicity) except one (the in vitro induction of [sister chromatid exchanges]), the proportion of chemicals predicted to be toxic among the HPV sample was significantly less than the proportion of chemicals predicted to be toxic in the [non-HPV] reference set". Likewise, the popular assumption that substantial "knowledge gaps" exist (1) does not withstand even a cursory review of the published literature for HPV chemicals (6-8). Had the EPA adhered to normal US government procedures and subjected the HPV programme to peer review and public comment, such pertinent information could have been used to objectively assess the true magnitude of the perceived problem and circumvent the massive animal testing programme now in place.

As a result of targeted campaigning by People for the Ethical Treatment of Animals (PETA), once the existence of the HPV programme became public knowledge, the US Government negotiated an agreement among the animal protection commu- 
nity, EPA, ACC, ED and the American Petroleum Institute (API), to incorporate certain minimal animal welfare measures into the HPV programme.

\section{Animal Welfare Guidance}

The basic components of the October 14, 1999, animal welfare agreement (9) are as follows:

\section{Avoiding checklists:}

In analysing the adequacy of existing data, participants shall conduct a thoughtful, qualitative analysis rather than use a rote checklist approach. Participants may conclude that there is sufficient data, given the totality of what is known about a chemical ... that certain endpoints need not be tested.

\section{Using existing data:}

Participants shall maximise the use of existing ... data to minimise further testing . . ..

\section{Using categories:}

Participants shall maximise the use of scientifically appropriate categories of related chemicals and structure-activity relationships.

\section{Testing only when relevant:}

Before generating new information, participants should further consider whether any additional information obtained would be useful or relevant.

\section{In vitro genotoxicity testing "encouraged":}

Participants are encouraged to use in vitro genetic toxicity testing . . . unless known chemical properties preclude its use.

\section{Analysis of Compliance}

A coalition of US animal protection organisations has reviewed all the 150 test plans submitted to date under the EPA's HPV programme, and has submitted comments on every one that has called for more animal testing, as well as on a number with no further proposed animal testing. (Note added in proof: as of August 2003, more than 220 test plans had been reviewed.) Approximately $75 \%$ of the submitted HPV test plans violated one or more fundamental aspects of the October 1999 animal welfare agreement (7). In so doing, numerous redundant, irrelevant, and useless animal tests have been proposed and conducted. In general, these tests fall into the following categories:

1. Testing without considering human exposure data.

2. Testing for secondary, irrelevant endpoints, when the primary hazard associated with the substance is high and well known.
3. Testing compounds with known or easily anticipated toxicities.

4. Testing for endpoints in a data rich environment, rather than analysing the universe of available data which would provide more insight into toxicity than the crude, screening level SIDS tests.

5. Testing without coordinating with other industries on similar substances.

6. Testing in vivo when in vitro tests are available.

The following are a few examples taken from the test plans and public responses posted on the EPA's HPV Web site (10).

\section{Failure to consider human use and exposure data}

The EPA consistently ignores human use and exposure data under the HPV programme, insisting instead that companies conduct tests on substances for which there is extensive past experience with human exposure through occupational, household or consumptive (food, water, air) exposure. Examples include additional testing of the following substances:

1. 1,3-Butadiene, even though it has been identified as a carcinogen, primarily through analysis of human exposure data from industrial settings.

2. Hydraulic fluids (such as trixylenyl phosphate), which are documented neurotoxicants and are therefore carefully managed substances. The toxicity of these substances is well documented in worker exposure data.

3. Many HPV substances are registered with the US Food and Drug Administration (FDA) as "Generally Recognised as Safe" (GRAS). These compounds generally have extensive databases demonstrating their lack of toxicity, and are found in many foods consumed each day. Yet Dupont, for example, proposed additional animal testing on adipic acid, which is GRAS and had already been found to be safe for use as a food additive in powdered drink mixes, puddings, and candies.

4. Many HPV substances are naturally occurring compounds, the biologic production and human metabolism of which are well understood, and which have an extensive history of human consumption. Yet, for example, The Pine Chemical Association (PCA) is conducting fish acute tox- 
icity and mammalian developmental toxicity testing on rosins and rosin salts, despite the fact that the material is insoluble, is a naturally occurring substance found primarily in pine trees, and is regulated by the FDA as a food additive, on which there is already extensive experience. Another example is the ionone derivative category, which are substances found mainly in plants containing $\beta$-carotene. Human exposure is more likely to occur via consumption of natural fruits, vegetables, and nuts than from products flavoured with the ionone derivatives. The original HPV test plan did not propose any animal testing; nonetheless the EPA called for additional developmental toxicity testing in its response to the test plan.

\section{Testing for irrelevant endpoints when the primary hazard is high and well known}

A number of companies have insisted on testing compounds with primary hazards which are already well known and well documented. However, if a compound is tightly regulated and exposure is extremely limited due to overwhelming obvious hazards, it makes little sense to conduct further crude, screening level studies on the compound.

The most obvious substances that fall into this category are explosive compounds. Many petroleum-derived and chemical feed stream gases are part of the HPV programme, including butane, 1,3 butadiene, methane, and isoprene, as well as complex streams containing these compounds and others. The ACC's test plan for crude butadiene proposed that acute toxicity testing be conducted on butadiene streams, and the API's test plan for petroleum gases proposed acute, repeat-dose, reproductive, and developmental toxicity studies on ethane, butane, propane, and isobutane. Existing data generally show no observed effect levels (NOELs) for these compounds at levels above 5\%; however, the lower explosive limit (LEL), the concentration at which a gas will explode when ignited, generally ranges between $1 \%$ and $4 \%$ for these gases. Furthermore, many of these gases have been studied as human anaesthetics in the past, and existing toxicity data show that they act primarily as simple asphixiants at levels exceeding $15 \%$. The proposals to conduct additional testing on such compounds ignore the most obvious hazard - their explosive nature - which already dictates how they are managed.

Many other compounds in the HPV programme are tightly regulated, and exposure to them is generally prevented, based on some specific toxicological properties of the compounds. For example, further screening-level testing has been proposed for a number of known carcinogens, despite the fact that human exposure is already extremely limited on the basis of existing knowledge of their more serious adverse effects. As mentioned above, 1,3 butadiene is regulated at levels less than $2 \mathrm{ppm}$ in industrial settings, but additional reproductive and subacute (repeat-dose) studies have been proposed to evaluate the hazard of several butadiene streams.

Many compounds in the HPV programme are also strong acids, bases, or oxidants, and are therefore extremely corrosive. For example, spent pulping liquor, a hydrogen sulphide-bearing strong base containing dissolved lignins and tannins, is obviously corrosive to any organism with which it comes into contact. Yet additional fish acute toxicity testing has been proposed and will be conducted. Because of the substance's high $\mathrm{pH}$, a neutralised form will be used for testing, thus rendering the results irrelevant to the starting material. A similar situation exists with cyclic anhydrides, which are unstable in water and immediately hydrolise to form strong acids. Even though these compounds are already well-known to be toxic, blinding, and corrosive, and are therefore tightly regulated with extremely limited exposures, the EPA endorsed the Industrial Health Foundation's plan to conduct a 90-day repeat-dose study and a reproductive and developmental toxicity study on these agents.

\section{Testing compounds with known toxicities}

Many substances in the HPV programme are mixtures of previously characterised compounds, the toxicities of which are already well known. Some proposed tests are unnecessary, since the answer can easily be anticipated and the results of the test will have little bearing on the ultimate regulation of the compound. For example, it is well known that hydrocarbons kill fish via non-polar necrosis, an effect that has little dependence on the specific structure of a compound. However, both the ACC and the EPA called for the acute aquatic testing of fuel oils, because there have been no tests of industrial streams with the same specific compositions. The additional tests will kill fish at similar levels to other hydrocarbons, but the checklist will be completed for the compound.

\section{Lack of contextual analysis}

Perhaps the greatest failing of the HPV programme is the failure to seriously examine existing data on related compounds (both HPV and non-HPV substances) prior to proposing testing. Because of the failure to develop appropriate categories and consider all existing data, information and understanding are lost. Moreover, when companies do take a broader view of the toxicity of substances by form- 
ing a category for the compounds, the EPA commonly rejects the category because it fails to meet the agency's narrow standard of analysis. To date, the EPA has objected to at least 27 of the 46 categories on which it has submitted comments. As a result, participants in the HPV programme are driven toward conducting more tests and simply "checking boxes", noting that all the SIDS endpoints are being fulfilled.

The process of analysing compounds in context is quite straightforward. Obvious, known hazards should be identified. Trends in similar compounds and their behaviours should be examined. The chemical and physical properties of the compounds should be understood before conducting new tests. Non-sensical endpoints, including chemically, physically, environmentally, and toxicologically irrelevant endpoints should not be assessed. Yet the PCA, for example, has repeatedly proposed conducting aquatic toxicity studies on compounds that are not soluble in water. The PCA has also proposed testing components of fish oils on fish, as in its test plan for tall oil fatty acids. A further example of an HPV test plan that completely fails to evaluate context is the plan to test trixylenyl phosphate. This compound is one of more than 20 phenyl phosphate compounds in the HPV programme, all of which are commonly used as antioxidants, stabilisers, and hydraulic fluids. However, the test plan's sponsor provides no information on the use and toxicity of this compound, and simply calls for testing of all SIDS endpoints, a plan with which the EPA concurred.

With poorly characterised compounds, it is senseless to conduct any toxicity tests until fundamental information on boiling point, water solubility, hydrolysis, vapour pressure, and $\mathrm{K}_{\mathrm{ow}}$ are known. In many cases, simply knowing the properties of a compound can preclude the need to conduct any further tests. Yet General Electric (GE), for example, submitted several test plans that failed to provide information on even these basic parameters and that nevertheless proposed extensive animal testing.

\section{Failure to coordinate across industries}

Different industries are not cooperating on test plans for similar substances, resulting in the performance of numerous redundant tests. For example, the petroleum coke test plan, submitted by the American Petroleum Institute, analyses two substances that are nearly identical in composition to carbon black (used in the rubber, plastics, and dye industries), anthracite coal (widely used as a fuel and having extensive human industrial exposure data), and coal coke (used in the steel industry). The extensive human data on exposure to these similar substances are not mentioned in the test plan, and the sponsors are planning to conduct additional reproductive and developmental toxicity studies on animals.

In its proposal to re-test tall oil fatty acids, the PCA failed to recognise that that many of the individual components of fatty acids, including palmitic acid, stearic acid, oleic acid, and linoleic acid, are being sponsored through the HPV programme by the Soap and Detergent Association. Clearly, these chemicals should have been combined into a single category under one HPV test plan. The lack of inter-industry cooperation leads directly to increased animal testing.

The API's test plan for the gasoline blending streams category proposed additional subacute (repeat-dose) and reproductive/developmental testing of streams high in naphthenes. The sponsor ignored the fact that benzene, toluene, and xylenes are the primary toxicants in gasoline streams, and that these compounds essentially drive all the hazard/risk assessments of the gasoline streams in the API's category. Abundant information exists on the potential hazards associated with streams containing naphthenes, and further testing will neither contribute to a meaningful increase in knowledge on the hazards of these compounds, nor change the manner in which they are regulated. The API also failed to expand its category to include the compounds in the ACC's low benzene naphtha category. As noted in the ACC's naphtha category test plan, many of the compounds in that category are also used in gasoline blending streams. Had the API maximised the use of chemical categories and coordinated across industries, it could have reduced the number of animals used and obtained more insight into the overall hazard associated with these compounds.

\section{Failure to use in vitro methods}

\section{Genetic toxicity}

The October 1999 agreement specifically calls for the use of internationally accepted in vitro tests for genotoxicity — which include the Ames/bacterial reverse-mutation test, as well as in vitro tests for chromosomal aberration, cell gene mutation, and sister chromatid exchanges (OECD Test Guidelines 471, 473, 476 and 479, respectively; 4). The use of these assays, alone or in combination, has been found to be highly sensitive to the detection of genotoxicants (11-12). Despite the scientific and ethical considerations favouring the use of in vitro methods for this endpoint, numerous in vivo genetic toxicity tests have been proposed by companies and endorsed by the EPA (10). The companies and organisations proposing to conduct the genetic tox- 
icity test in vivo include GE, FMC Corporation, ExxonMobil, the PCA, the API, and the ACC.

\section{Acute fish ecotoxicity}

To date, the EPA continues to reject the use of viable in vitro assays for the assessment of acute toxicity to fish (10). One alternative that has been repeatedly proposed is the TETRATOX assay, which uses the protozoan, Tetrahymena, as a biomarker for aquatic toxicity in ecological risk assessments (13). The biochemistry and physiology of Tetrahymena have been thoroughly investigated since the $1950 \mathrm{~s}$, and Tetrahymena, and especially $T$. pyriformis, have been used for aquatic toxicity testing since the 1970s. Moreover, the genomics of the organism are currently being elucidated. The $T$. pyriformis population growth test is rapid, inexpensive and has considerable breadth. Data generated by the TETRATOX assay demonstrate a consistently high degree of concordance to data from acute toxicity studies in fish.

Another promising in vitro assay is a fish egg test (14), which exposes very early developmental stage embryos to concentrations of a test substance. This test has recently been accepted in Germany as a replacement for the use of fish in the assessment of waste-water effluent, and is being proposed as a total replacement to all uses of fish in acute ecotoxicity studies.

In addition, ECOSAR (ecological structure-activity relationships), an in silico model used to predict chemical toxicity to aquatic organisms, has been vastly underused in the HPV programme, despite explicit guidance from the EPA (15). The model is capable of predicting effects on a range of taxonomic groups, including fish, invertebrates, and algae, as well as effects following both acute and chronic exposure (16).

\section{Developmental toxicity}

An embryonic stem cell test (EST) has recently been validated by the European Centre for the Validation of Alternative Methods (ECVAM) as a test for embryotoxicity - a critical parameter and manifestation of developmental toxicity (17). The test uses rodent-derived stem cells, which survive in culture indefinitely and are capable of differentiation. Embryotoxicity is determined by the concentration of a test chemical required to inhibit differentiation by $50 \%$, together with growth inhibition by $50 \%$ relative to controls. This validated test method is ideally suited for immediate use as a reduction measure in a basic, screening-level programme like the EPA's HPV Challenge - whereby chemicals that test positive for embryotoxicity could be classified as probable developmental toxi- cants without further testing. Despite its enormous potential as a reduction measure - which would spare the lives of the more than 1300 animals used in a standard prenatal developmental toxicity study according to OECD 414 (4) - the EPA and the participating companies have refused to make use of this test under the HPV programme (10).

\section{Acute mammalian toxicity}

Participants at an October 2000 international workshop on in vitro methods for assessing acute systemic toxicity concluded that two basal cytotoxicity assays using normal human keratinocytes (NHK) and mouse fibroblast (Balb/c 3T3) cell lines were suitable for immediate use as a reduction measure to calculate starting doses in vivo (18-19). Spielmann and colleagues (20) have reported that their use has the potential to reduce animal use by up to $40 \%$. The EPA subsequently issued guidance to HPV programme participants (21) urging them to use this strategy in the generation of acute toxicity data under this programme. However, companies proposing to conduct acute toxicity testing have frequently ignored the recommended use of in vitro cytotoxicity assays as a dose-setting measure - even those proposing to test known non-toxic materials (10). To date, the EPA has taken no remedial action in this matter.

\section{Toll in Animal Lives}

A review, completed in October 2002, summarised all the animal tests proposed to date in HPV test plans, as well as those requested by the EPA over and above those proposals. Calculations based on the OECD Guidelines for the Testing of Chemicals (4) suggest that the total number of animals already killed in the US HPV programme is approximately 100,000. Whilst the EPA continues to claim that few new animal tests are being conducted under the HPV programme (22), this is clearly not the case, as fully half of all the test plans submitted proposed to conduct additional animal testing. Our conservative estimate found that more than 250 new animal tests have been conducted to date, resulting in the wasting of this inordinately high number of animal lives. Because the HPV programme began with category testing and data-rich compounds, the number of animals killed as the programme continues can also be expected to climb dramatically.

\section{Conclusions}

The US EPA is ignoring the 1999 animal welfare agreement and has repeatedly encouraged a check- 
the-box approach to testing under the HPV programme. Even though a thorough analysis of existing data would be adequate for ranking the hazards of many of the HPV compounds, companies propose the performance of numerous irrelevant tests to satisfy EPA demands. The exclusion of human use and exposure experience, the insistence on testing for all endpoints (including for GRAS chemicals), and the failure to approve proposed category testing, have led to testing for endpoints that are chemically, physically, and/or toxicologically irrelevant. In vitro screening tools are being ignored, whilst tests that are not a part of the HPV programme are repeatedly proposed (e.g. skin and eye irritation, chronic fish and terrestrial ecotoxicity, 90-day repeat-dose tests, and studies via the dermal exposure route).

The EPA has clearly exhibited a double standard with regard to animal testing in its responses to proposed HPV test plans. In general, the EPA presumes that more animal testing is required. No justification is required if a company plans to use animal tests - even when the company has clearly ignored existing data - whilst there is disproportionate scrutiny of all proposals to use categories, structure-activity relationship analyses or existing data, in order to avoid further testing on animals. The EPA ignores the comments submitted by the animal protection community and does not require adherence to the most basic animal welfare principles. In a number of instances, the EPA has posted its own comments prior to the deadline for public comments, thus ensuring that public comments are not taken into consideration.

The US HPV programme lacks a contextual framework, which is necessary in order to prioritise and understand toxicity and chemical hazards. It defaults to a check-the-box approach, which simply ensures compliance with bureaucratic mandates, but does very little to protect public health and the environment. Its reliance on a simple checklist approach leads to overconfidence in hazard assessment instead of demanding a serious review of existing physical, chemical, exposure and toxicological information. Conclusions may be reached based on irrelevant tests or tests which are essentially worthless in extrapolating to humans, due to dramatic interspecies variability, lack of consideration of exposure pathways, or lack of understanding of the fundamental properties of a compound. The HPV programme is therefore resulting in a large number of animal deaths for precious little useful information.

\section{Acknowledgements}

The following organisations contribute to the ongoing test plan review with either expertise and/or financial support: People for the Ethical Treatment of Animals; The Physicians Committee for Responsible Medicine; The Doris Day Animal League; The Humane Society of the United States; and Earth Island Institute.

\section{References}

1. Silbergeld, E.D. (1998). Public comments at the November 1998 HPV stakeholders' meeting, Washington, DC, USA.

2. Hernandez, O. (1998). Public comments at the November 1998 HPV stakeholders' meeting, Washington, DC, USA.

3. Organisation for Economic Cooperation and Development. (1999). Synopsis of OECD Test Guidelines for Studies included in the SIDS. Web site http://www1.oecd.org/ehs/guide/. Paris, France: OECD (Accessed 8.12.02).

4. Organisation for Economic Cooperation and Development (2002). OECD Guidelines for the Testing of Chemicals. Paris, France: OECD.

5. Cunningham, A.R. \& Rosenkranz, H.S. (2001). Estimating the extent of health hazard posed by high production volume chemicals. Environmental Health Perspectives 109, 953-956.

6. Physicians Committee for Responsible Medicine (1998). Availability of HPV Chemical Data, 40pp. Washington, DC, USA: PCRM.

7. Cardello, N. (2001). Analysis of the HPV Challenge Programme: Industry Violations and EPA Negligence, 17pp. Washington, DC, USA: PCRM.

8. Spitzer, H. \& Wilson, J. (1997). Toxic ignorance or toxic terror. Risk Policy Report 4, 32.

9. Wayland, S. (1999). Letter to HPV programme participants, 2pp. Web site http://www.epa.gov/chemrtk/ ceoltr2.htm. Washington, DC, USA: US EPA.

10. US Environmental Protection Agency (2002). Chemical Right to Know. Web site http://www.epa.gov/ chemrtk/viewsrch.htm. Washington, DC, USA: US Environmental Protection Agency (Accessed 8.12.02)

11. Zeiger, E. (1987). Carcinogenicity of mutagens: predictive capability of the Salmonella mutagenesis assay for rodent carcinogenicity. Cancer Research 47, 1287-1296

12. Zhang, Y.P., Van Praagh, A., Klopman, G. \& Rosenkranz, H.S. (1994). Structural basis of the induction of unscheduled DNA synthesis in rat hepatocytes. Mutagenesis 9, 141-149.

13. Sinks, G.D. \& Schultz, T.W. (2001). Correlation of Tetrahymena and Pimephales toxicity: evaluation of 100 additional compounds. Environmental Toxicology and Chemistry 20, 917-921.

14. Schulte, C. \& Nagel, R. (1994). Testing acute toxicity in the embryo of zebrafish, Brachydanio rerio, as an alternative to the acute fish test: preliminary results. ATLA 22, 12-19.

15. US Environmental Protection Agency (2000). The Use of Structure-Activity Relationships (SAR) in the High Production Volume Chemical Challenge Programme. Washington, DC, USA: US Environmental Protection Agency (Accessed 8.12.02).

16. US Environmental Protection Agency (2002). Ecological Structure Activity Relationships. Web site http://www.epa.gov/opptintr/newchems/21ecosar.htm. Washington, DC, USA: EPA (Accessed 8.12.02). 
17. Genschow, E., Spielmann, H., Scholz, G., Seiler, A., Brown, N., Piersma, A., Brady, M., Clemann, N., Huuskonen, H., Paillard, F., Bremer, S. \& Becker, K. (2002). The ECVAM international validation study on in vitro embryotoxicity tests: results of the definitive phase and evaluation of prediction models. ATLA 30, 151-176.

18. US National Toxicology Program Interagency Committee for the Evaluation of Alternative Toxicological Methods (2001). Report of the International Workshop on In Vitro Methods for Assessing Acute Systemic Toxicity, 389pp. NIH Publication 01-4499. Web site http://iccvam. niehs.nih.gov/methods/invidocs/guidance/iv_guide. htm. Research Triangle Park, NC, USA: National Institute of Environmental Health Sciences (Accessed 8.10.02).

19. Liebsch, M., Curren, R. \& Fentem, J. (2001). Guidance Document on Using In Vitro Data to Estimate In Vivo
Starting Doses for Acute Toxicity. NIH Publication 014500. Web site http://iccvam.niehs.nih.gov/methods/ invidocs/guidance/iv_guide.pdf. Research Triangle Park, NC, USA: National Institute of Environmental Health Sciences (Accessed 8.10.02).

20. Spielmann, H., Genschow, E., Liebsch, M. \& Halle, W. (1999). Determination of the starting dose for acute oral toxicity (LD50) testing in the Up and Down Procedure (UDP) from cytotoxicity data. ATLA 27, 957-966.

21. US Environmental Protection Agency (2001). Supplemental Acute Toxicity Protocol. Web site http://www.epa.gov/chemrtk/toxprtcl.htm. Washington, DC, USA: US Environmental Protection Agency (Accessed 8.12.02).

22. US Environmental Protection Agency (2001). Status Report on the HPV Challenge Programme, 46pp. Washington, DC, USA: US Environmental Protection Agency. 